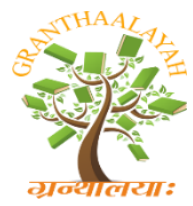

Science

\title{
CAN INCREASED MONOCYTE / LYMPHOCYTE RATIO BE USED AS AN INDICATOR FOR ALZHEIMER'S DISEASE?
}

\author{
Aslı Bolayır *1 \\ ${ }^{* 1}$ Assistant Professor, Cumhuriyet University Medical Faculty,Neurology Department, Sivas, \\ Turkey
}

\begin{abstract}
Background: The monocyte to lymphocyte ratio (MLR) obtained by dividing the number of monocytes by lymphocyte number is considered as a new marker of inflammation. The role of MLR in Alzheimer's disease (AD) has not been clearly understood yet. So, the aim of this study is to determine the role of MLR in the pathogenesis of AD.

Method: Our study is a single centered retrospective study. 254 patients with AD, who applied to our clinic between January 2013 and December 2017 and 256 healthy control with similar age and sex were included to this study.

Results: While lymphocyte count was lower in the control group; MLR values were higher in the patient group. The patient group was then divided into three subgroups according to the results of standardized mini mental test (SMMT). MLR values increased in parallel with the severity of AD. In addition, ROC analysis revealed that MLR values above 0.28 can be used as a marker of the development of AD (AUC: 0.76, 95\% CI: 0.66-0.87, sensitivity: 73.1\%, specificity of 81.1\%).

Conclusions: This study demonstrated the role of MLR in the inflammatory process, thought to be responsible for the development of AD.

Keywords: Dementia; Monocyte; Lymphocyte; Monocyte/Lymphocyte Ratio; Alzheimer's Disease.

Cite This Article: Aslı Bolayır. (2018). "CAN INCREASED MONOCYTE / LYMPHOCYTE RATIO BE USED AS AN INDICATOR FOR ALZHEIMER'S DISEASE?." International Journal of Research - Granthaalayah, 6(4), 198-204.
\end{abstract}

\section{Introduction}

Alzheimer's disease (AD), known since 1907, is a serious neurodegenerative disease and the most common form of senile dementia affecting about 45 million people worldwide (1). AD is a syndrome characterized by progressive deterioration in cognitive functions and behavior (2). The increasing prevalence of $\mathrm{AD}$, threaten seriously the elderly population and the economy that supports them. More effective treatment strategies for AD should be developed to reduce this massive psychosocial and public health burden. This is only possible with a beter understanding of the pathophysiology of the disease. 
One of the principal neuropathological alterations in $\mathrm{AD}$ is amyloid plaques, whose main component is amyloid beta protein (A $\beta)$. Amyloid $\beta$ aggregates as diffuse plaques and these turn into dense neuritic plaques. It is unclear how loose plaques containing harmless amyloid $\beta$ are transformed into beta-curved harmful dense plaques. Amyloid $\beta$ accumulation in loose plaques may lead to oxidative stress and the formation of the free radicals, which may lead to physical changes of plaques (4). After development of the neuritic plaques, secondary cascade, including inflammation, excitotoxicity and possibly apoptosis mediates additional damage. Microglia, which mainly produce inflammatory cytokines, astrocytes and neurons play a major role in the inflammatory process of AD.

The presence of proinflammatory cytokines in both the central nervous system and periphery was detected in AD (5). These findings suggest that both innate and acquired immunological responses are effective in the disease process. Circulating peripheral cells such as leukocytes, lymphocytes and macrophages are mentioned to be used as an indicator of systemic inflammation in the literature (6). In addition, some studies showed that the count of peripheral leukocyte in patients with AD could vary according to the healthy controls (7).

Microglial cells, come from the monocyte-macrophage family, are one of the well-known cell type in the central nervous system. Peripheral monocytes, from the same family as the microglial cells, are involved in the immune response by a variety of cell death ligands and cytokines, which exfoliate on the surface of monocytes and increase in inflammatory conditions $(6,7)$.

The neutrophil to lymphocyte ratio (NLR) associated with cerebro/cardiovascular diseases is considered a new indicator of systemic inflammation. Recent studies have shown the alteration of neutrophil and lymphocyte counts and the elevation of NLR values in AD compared with similar age-matched healthy controls (8). The monocyte to lymphocyte ratio (MLR) is accepted as an another newly identified inflammatory marker. The relationship between MLR values and various malignancy and cerebrovascular disease was revealed (9-11). However, the role of MLR in AD has not been clearly understood yet.

So, we aimed in this retrospective study to demonstrate the role of the monocyte count and MLR values in the inflammatory process that is thought to be responsible for pathophysiology of AD by comparing the monocyte counts and MLR values of the AD patients with healthy controls.

\section{Methodology}

\section{The creation of the study population:}

This study is a single-center retrospective study that included 254 patients older than 65 years who admitted to Cumhuriyet University Neurology Department with a complaint of dementia and were diagnosed with AD between January 2013 and December 2017. The medical history, cranial magnetic resonance or computed tomography imaging findings showed no additional pathology (chronic subdural hematoma, intracranial mass...) except cerebral atrophy, normal results of laboratory tests to exclude additional diseases, the neurological examination findings and standardized mini mental test(SMMT) results of patients were used in order to determine the definitive diagnosis of AD. There were no restrictions regarding sex and age among the patients. Exclusion criteria were systematic acute/chronic infectious/inflammatory/autoimmune or 
hematological diseases, cancer, severe liver, kidney or heart failure, acute coronary syndrome/ acute myocardial infarction/cerebrovascular diseases within the past three months, prior a history of major surgery or trauma, and using immunosuppressant and antiinflammatory drugs or steroids. The patient group was divided into three subgroups according to SMMT results (1. group: SMMT 20-23; 2. group: SMMT: 10-19; 3. group: SMMT: 0-9).

The control group consisted of 256 patients with no complaint about dementia who were age-and gender-matched, did not suffer from any uncontrolled systemic diseases such as diabetes mellitus, hypertension, cardiovascular diseases or cancer, and gave blood samples for reasons other than AD between January 2013 and December 2017.

All patients included in the current study were examined and investigated in terms of parameters such as age, gender, diabetes mellitus (defined as the fasting blood glucose level above $126 \mathrm{mg} / \mathrm{dl}$ and/or the use of antidiabetic drugs), hypertension (defined as the systolic blood pressure above $140 \mathrm{mmHg}$ and/or the diastolic blood pressure above $90 \mathrm{mmHg}$, or the use of antihypertensive treatment), hyperlipidemia, tobacco or statin use, plasmaglucose, creatinine, C-reactive protein (CRP) levels, hemoglobin, monocyte, lymphocyte, neutrophil and white blood cell (WBC) counts, MLR values and SMMT results.

The missing information in our retrospective study was obtained via telephone interview with patients or their relatives.

Approval by the ethics committee of Cumhuriyet University was obtained for this study.

\section{Evaluation of biochemical and hematological parameters:}

Blood samples of all patients and controls included in the study were taken at the right antecubital vena in rest position. While dry tubes were used for the biochemical analysis, for the hematological test, EDTA tubes were utilized. Whole blood counts of both groups were evaluated with a Mindray BC- 6800 device using Diagon Kits. The monocyte and lymphocyte counts of patients and controls were obtained from the results of this device. The MLR values were calculated by dividing the number of monocytes by the number of lymphocytes. Biochemical analyses were performed with a Beckman Coulter AU5800 device (Beckman Coulter Inc, Hialeah, Florida) using kits produced by the same company via a fully automatic nephelometric method.

\section{Statistical analysis:}

Data obtained from this study was evaluated by using the SPSS 22.0 program. The KolmogorovSmirnov test was used to determine whetherthe data were normally distributed. While the normally distributed continuous data were expressed as the mean \pm Standard deviation, the non-normally distributed continuous data were expressed as median (min-max). The categorical data were indicated as percentage (\%). While the independent T-test was used for the analysis of continuous data with normal distribution, the Mann-Whitney U-test was used for the analysis of continuous variables not showing normal distribution. The comparision of the categorical datas was evaluated by the chi squared test. The Kruskal-Wallis $\mathrm{H}$ test was used to compare the data of the patient group's subgroups. Receiver operating characteristic curve (ROC) analysis was used for determining the optimum cut-off value of MLR for predicting AD development. The statistical significance level was accepted as $\mathrm{p}<0.05$. 


\section{Findings}

Comparison of the patient and control groups in terms of baseline demographic characteristics showed that there was no significant difference between the two groups in terms of age, gender, the presence of diabetes mellitus, hypertension or hyperlipidemia and tobacco or statin use. The mean age of the patients was $74.22 \pm 6.2$, while the control group was $73.01 \pm 6.2$ years. The comparison of the laboratory parameters revealed no significant difference between the two groups in terms of glucose, creatinine, hemoglobin levels and $\mathrm{WBC}$, neutrophil and monocyte counts $(\mathrm{p}=0.31, \mathrm{p}=0.12, \mathrm{p}=0.49, \mathrm{p}=0.43, \mathrm{p}=0.14, \mathrm{p}=0.12$ and $\mathrm{p}=0.23$, respectively). Besides, there were significant differences between the two groups in terms of CRP, monocyte, lymphocyte counts and MLR values. While CRP and MLR values were higher in the patient group, the lymphocyte count was significantly higher in the control group (Table 1). The patient group was divided into three subgroups according to the SMMT results. The SMMT results of the the first group were between 20-23 $(\mathrm{n}=94)$; the second group's SMMT results were between 10-19 $(\mathrm{n}=$ 136); and the SMMT results of the patients in the third group were between 0-9 $(n=24)$. The comparison of these three group in terms of lymphocyte count and CRP and MLR values showed that the lymphocyte counts were not different between the three groups, while CRP and MLR values were the lowest in the first and the highest in third group and there was a statistically significant difference between the three groups for these two values $(\mathrm{p}=0.04, \mathrm{p}=0.03$, respectively) (Table 2).

Additionally, the receiver operating characteristic curve (ROC) analysis showed that the values above 0.28 for MLR can be used with high sensitivity and specificity in detecting AD development (the area under the curve (AUC) value: $0.76,95 \%$ confidence interval (CI) 0.66-0.87, sensitivity: $73.1 \%$, specificity of $81.1 \%)$.

Table 1: The comparison of the baseline demographic, clinical and laboratory characteristics of the control and the patients groups.

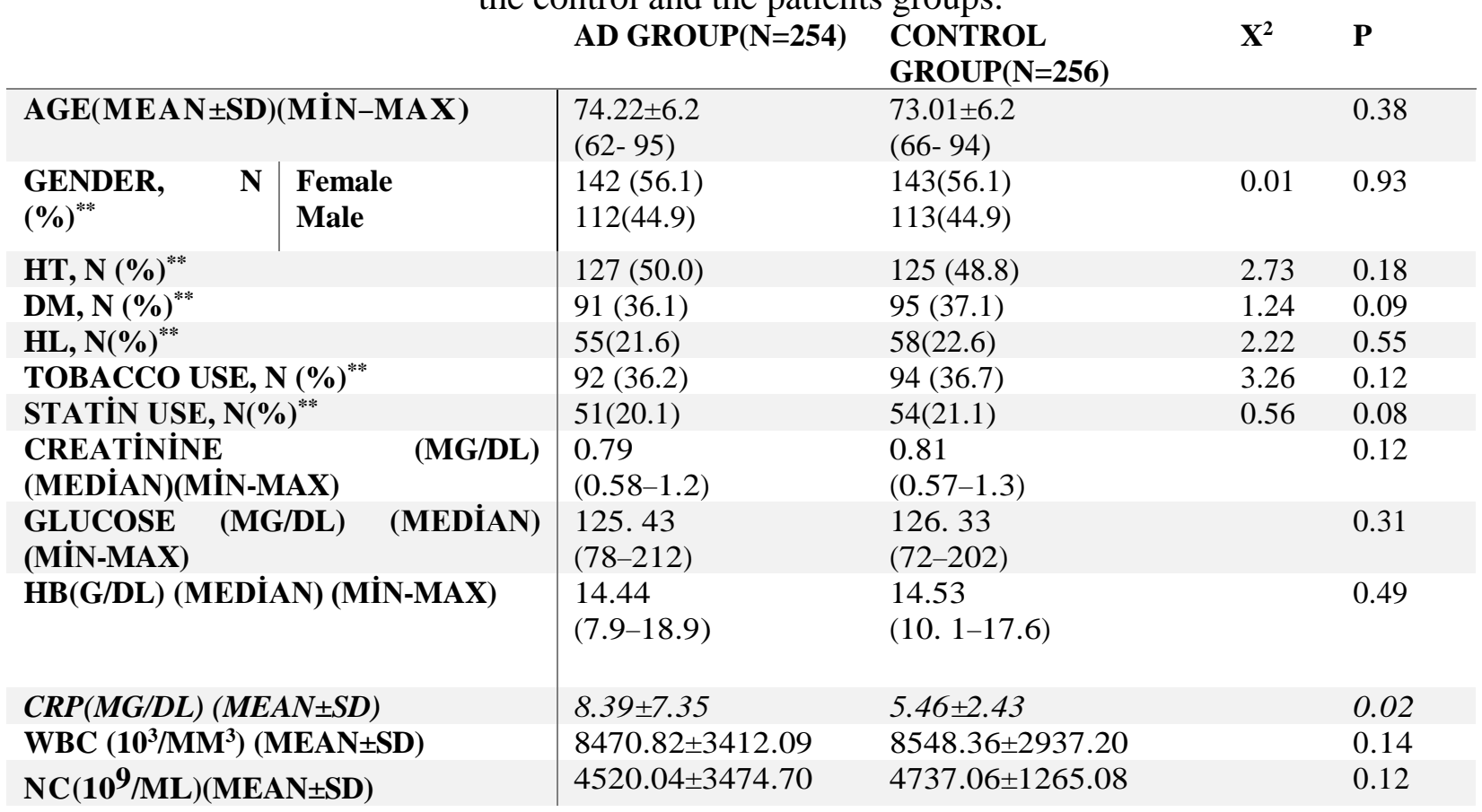




\begin{tabular}{|c|c|c|c|}
\hline $\mathrm{MC}\left(10^{9} / \mathrm{ML}\right)(\mathrm{MEAN} \pm \mathrm{SD})$ & $414.04 \pm 61.34$ & $411.49 \pm 43.20$ & 0.23 \\
\hline$L C\left(10^{9} / M L\right)(M E A N \pm S D)$ & $1250.63 \pm 705.29$ & $1797.28 \pm 520.08$ & 0.04 \\
\hline$M L R(M E D \dot{I} A M)(M \dot{I}-M A X)$ & $\begin{array}{l}0.32 \\
(0.22-0.54)\end{array}$ & $\begin{array}{l}0.23 \\
(0.15-0.34)\end{array}$ & 0.02 \\
\hline
\end{tabular}

Italic values are used to demonstrate the statistical significance. **: Chi square test used. Abbreviations: CRP: Creactive protein, DM: diabetes mellitus, Hb: hemoglobin, HT: hypertension, MC: monocyte count, MLR: monocyte to lymphocyte ratio, NC: neutrophil count, LC: lymphocyte count, TotalCho: total cholestrol, SD: stamdard deviation, WBC: white blood cell.

Table 2: The comparison of the mean lymphocyte counts and LMR and CRP values in patient group after classification into SMMT results.

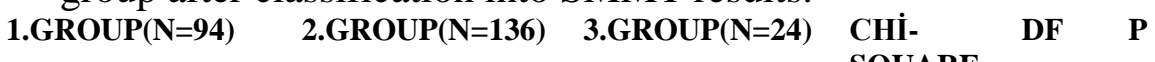

\begin{tabular}{|c|c|c|c|c|c|c|}
\hline $\begin{array}{l}\text { CRP(MG/DL) } \\
(M E A N \pm S D)\end{array}$ & $7.24 \pm 2.42$ & $8.93 \pm 3.16$ & $9.75 \pm 5.36$ & 9.22 & 2 & 0.04 \\
\hline $\begin{array}{l}\mathrm{LC}\left(\mathbf{1 0}^{9} / \mathrm{ML}\right) \\
(\mathrm{MEAN} \pm \mathrm{SD})\end{array}$ & $1254.98 \pm 220.54$ & $1247.01 \pm 520.14$ & $1248.04 \pm 720.21$ & 11.98 & 2 & 0.12 \\
\hline $\begin{array}{l}\text { MLR(MEDİAN) } \\
\text { (Mİ-MAX) }\end{array}$ & $0.25(0.22-0.35)$ & $0.35(0.27-0.49)$ & $0.39(0.39-0.54)$ & 4.22 & 2 & 0.03 \\
\hline
\end{tabular}

Abbreviations: CRP: C- reactive protein, SMMT: standardized mini mental test, MLR: monocyte to lymphocyte ratio, LC: lymphocyte count, SD: stamdard deviation.

\section{Discussions}

This study showed that the patients with AD had higher MLR values than age- matched healthy controls. In addition, it was also found that there was a positive correlation between the level of MLR, considered as a new inflammatory marker, and the severity of AD.

The role of the innate and acquired immunity system in the central nervous system is getting better understood in recent times. Increasing evidences suggest that there is a relationship between brain tissue and defective immunity in AD. The recent studies have shown that the peripheral monocyte count changes in patients with AD (6). In contrast, in our study, there was no difference in monocyte count between the patient group and the control group and between the subgroups of the patient group. Some studies, which was supported by our results, showed that, there was no differences in monocyte count in patients with $\mathrm{AD}$ but the monocytes' poor differentiation into macrophages resulted in impaired $\mathrm{A} \beta$ phagocytosis in AD (12). Additionally, it is known that peripheral blood derived monocytes can infiltrate the brain tissue and remove cerebral A $\beta$ deposits more effectively than resident microglia to preserve tissue homeostasis (13). However, it was revealed that there was an age-related dysfunction in migration and infiltration of peripheral monocytes into brain tissue, which may explain the absence of differences in the monocyte count in our study (14).

Recently, it has been revelaed that, the changes of lymphocyte count, subtypes of leukocytes, is associated with several neurological diseases $(8,15)$. Lymphocytes have a controversial role in the inflammation. It was showed, that elevated lymphocytes increase anti-inflammatory cytokine, interleukin (IL)-10, and suppress inflammatory cytokines, such as IL-6 and tumor necrosis factor (TNF)- $\alpha$, stimulating neuroprotective effect (14). Shortly, lymphocytes have beneficial roles in 
suppressing inflammation. It is known that the $\mathrm{A} \beta$ deposits trigger inflammation in $\mathrm{AD}$. In our study, higher CRP levels were detected in the patient group when there were lower lymphocyte counts compared to the control group. This finding is also compatible with the studies showing increased inflammation in $\operatorname{AD}(5,6,16)$.

MLR, which is obtained by dividing the number of monocytes by the number of lymphocytes, is regarded as a new inflammatory indicator and its association with many neurological diseases is known. In our study, higher MLR values were detected in the patient group compared to the control group. In addition, it was found that both CRP, known as a good marker of inflammation and MLR values increased in parallel with the severity of $\mathrm{AD}$, although the lymphocyte counts did not change(Table 2). Based on these results; it can be claimed that MLR values is superior to both monocyte and lymphocyte counts in terms of both the development and severity of AD. Besides, it was also been showed that MLR values above 0.28 have high sensitivity and specificity in detecting AD development.

There are several limitations of this study. Firstly, this study is a retrospective, one-centered study. So, the number of patients is relatively small. In addition, other inflammatory markers such as IL-10, TNF- $\alpha$ were not measured in patient and control groups.

\section{Conclusions}

In conclusion, this study demonstrated the role of MLR, which is easily accessible and therefore does not require additional expense, in the inflammatory process, thought to be responsible for the development of AD. More detailed and extensive multicenter prospective studies are needed to assess our results.

\section{No acknowledgement and financial support was declared.}

\section{All of the authors declared no conflict of interest.}

\section{References}

[1] Prince, M. J. (2015). World Alzheimer Report 2015: the global impact of dementia: an analysis of prevalence, incidence, cost and trends. Alzheimer's Disease International.

[2] Gürvit, H.(2014). Sinir Sisteminin Dejeneratif Hastalıkları. Demans Sendromu, Alzheimer Hastalığı ve Alzheimer dışı demanslar. In: Öge AE; eds. Nöroloji. İstanbul: Nobel Matbaacılık: 367- 415.

[3] Glenner, G. G., \& Wong, C. W. (1984). Alzheimer's disease: initial report of the purification and characterization of a novel cerebrovascular amyloid protein. Biochemical and biophysical research communications, 120(3), 885-890.

[4] Bird, T. D. (2001). Alzheimer's disease and other primary dementias. Harrisons principles of internal medicine, 2, 2391-2398.

[5] Lee, K. S., Chung, J. H., Choi, T. K.,et al. (2009). Peripheral cytokines and chemokines in Alzheimer's disease. Dementia and geriatric cognitive disorders, 28(4), 281-287.

[6] Wilson, E. H., Weninger, W., \& Hunter, C. A. (2010). Trafficking of immune cells in the central nervous system. The Journal of clinical investigation, 120(5), 1368-1379. 
[7] Imtiaz, F., Shafique, K., Mirza, S. S., et al (2012). Neutrophil lymphocyte ratio as a measure of systemic inflammation in prevalent chronic diseases in Asian population. International archives of medicine, 5(1), 2 ..

[8] Kuyumcu, M. E., Yesil, Y., Oztürk, Z. A., et al. (2012). The evaluation of neutrophil-lymphocyte ratio in Alzheimer's disease. Dementia and geriatric cognitive disorders, 34(2), 69-74.

[9] Song, W., Tian, C., Wang, K., et al. (2017). The pretreatment lymphocyte to monocyte ratio predicts clinical outcome for patients with hepatocellular carcinoma: A meta-analysis. Scientific Reports, 7, 46601.

[10] Ren, H., Liu, X., Wang, L., \& Gao, Y. (2017). Lymphocyte-to-monocyte ratio: A novel predictor of the prognosis of acute ischemic stroke. Journal of Stroke and Cerebrovascular Diseases, 26(11), 2595-2602.

[11] Ren, H., Han, L., Liu, H., et al. (2017). Decreased Lymphocyte-to-Monocyte Ratio Predicts Poor Prognosis of Acute Ischemic Stroke Treated with Thrombolysis. Medical science monitor: international medical journal of experimental and clinical research, 23, 5826.

[12] Fiala, M., Liu, Q. N., Sayre, J., et al. (2002). Cyclooxygenase-2-positive macrophages infiltrate the Alzheimer's disease brain and damage the blood-brain barrier. European journal of clinical investigation, 32(5), 360-371.

[13] Simard, A. R., Soulet, D., Gowing, G., et al. (2006). Bone marrow-derived microglia play a critical role in restricting senile plaque formation in Alzheimer's disease. Neuron, 49(4), 489-502.

[14] Malavolta, M., Basso, A., Piacenza, F., et al. (2013). Peripheral mononuclear cell rejuvenation for senescence surveillance in Alzheimer disease. Current pharmaceutical design, 19(9), 1720-1726.

[15] Bolayır, A., \& Gökçe, Ş. F. (2017). The role of mean platelet volume, platelet distribution width and platelet/lymphocyte ratio in development of cerebral venous thrombosis. Cumhuriyet Medical Journal, 39(4), 683-691.

[16] Enciu, A. M., \& Popescu, B. O. (2013). Is there a causal link between inflammation and dementia?. BioMed research international, 2013.

*Corresponding author.

E-mail address: asliarslanturk@ gmail.com 